\title{
OPEN A scenario modeling pipeline for COVID-19 emergency planning
}

\author{
Joseph C. Lemaitre ${ }^{1,8}$, Kyra H. Grantz ${ }^{2,8}$, Joshua Kaminsky ${ }^{2,8}$, Hannah R. Meredith ${ }^{2,8}$, \\ Shaun A. Truelove ${ }^{3,4,8}$, Stephen A. Lauer ${ }^{2}$, Lindsay T. Keegan ${ }^{5}$, Sam Shah ${ }^{6}$, Josh Wills, \\ Kathryn Kaminsky ${ }^{7}$, Javier Perez-Saez ${ }^{2}$, Justin Lessler ${ }^{2}$ \& Elizabeth C. Lee ${ }^{2 \bowtie}$
}

Coronavirus disease 2019 (COVID-19) has caused strain on health systems worldwide due to its high mortality rate and the large portion of cases requiring critical care and mechanical ventilation. During these uncertain times, public health decision makers, from city health departments to federal agencies, sought the use of epidemiological models for decision support in allocating resources, developing non-pharmaceutical interventions, and characterizing the dynamics of COVID-19 in their jurisdictions. In response, we developed a flexible scenario modeling pipeline that could quickly tailor models for decision makers seeking to compare projections of epidemic trajectories and healthcare impacts from multiple intervention scenarios in different locations. Here, we present the components and configurable features of the COVID Scenario Pipeline, with a vignette detailing its current use. We also present model limitations and active areas of development to meet ever-changing decision maker needs.

In late 2019, the virus responsible for coronavirus disease 2019 (COVID-19) was detected in Wuhan, China ${ }^{1}$. Since its emergence, SARS-CoV-2 has spread rapidly, causing significant morbidity and mortality; prompting the World Health Organization to declare a pandemic on 11 March $2020^{2}$. In addition to its significant individual health impacts, COVID-19 has put considerable strain on health systems, as a large fraction of cases require mechanical ventilation or critical care ${ }^{3}$. In every stage of the pandemic thus far, there has been a need for flexible decision support tools that can be used to model and compare critical planning scenarios.

Epidemiological models have played an important role in shaping public health policy and interventions throughout the pandemic. The methods used have ranged widely-from agent-based modeling approaches that simulate the global movement of individuals and their contacts in household, workplace, and leisure settings ${ }^{4}$, to population-level models that incorporate features like age-specific transmission, asymptomatic and presymptomatic transmission, and metapopulation structure ${ }^{5-7}$, to curve fitting approaches that use data from early in the COVID-19 pandemic to project future burden ${ }^{8}$. Likewise, the goals of these models have varied widely, from assessing importation risk, estimating the fraction of cases attributable to transmission from unobserved infections, projecting the impact of non-pharmaceutical interventions that target different populations, and forecasting the needs of the healthcare system.

Within this space, there was a need for a modeling pipeline that could provide flexible but sophisticated epidemiological models to decision makers who needed to plan and compare specific interventions. Here, we detail our scenario modeling pipeline, a modular framework that projects epidemic trajectories and health care impacts under different suites of interventions in order to aid in scenario planning. The flexibility of our approach has allowed us to provide rapid support to multiple organizations at the same time, while customizing our models to situation-specific questions and data. This framework has been used to provide tailored estimates of the relative impacts of different scenarios of disease transmission, severity, and control, thus guiding intervention policies in several states, countries, and humanitarian aid settings.

\footnotetext{
${ }^{1}$ Laboratory of Ecohydrology, School of Architecture, Civil and Environmental Engineering, École Polytechnique Fédérale de Lausanne, Lausanne, Switzerland. ${ }^{2}$ Department of Epidemiology, Johns Hopkins Bloomberg School of Public Health, Baltimore, MD, USA. ${ }^{3}$ Department of International Health, Johns Hopkins Bloomberg School of Public Health, Baltimore, MD, USA. ${ }^{4}$ International Vaccine Access Center, Johns Hopkins Bloomberg School of Public Health, Baltimore, MD, USA. ${ }^{5}$ Division of Epidemiology, Department of Internal Medicine, University of Utah, Salt Lake City, UT, USA. ${ }^{6}$ Unaffiliated, San Francisco, USA. ${ }^{7}$ Unaffiliated, Baltimore, USA. ${ }^{8}$ These authors contributed equally: Joseph C. Lemaitre, Kyra H. Grantz, Joshua Kaminsky, Hannah R. Meredith and Shaun A. Truelove. ${ }^{\varpi}$ email: elizabeth.c.lee@jhu.edu
} 
Feedback from health departments and policy makers

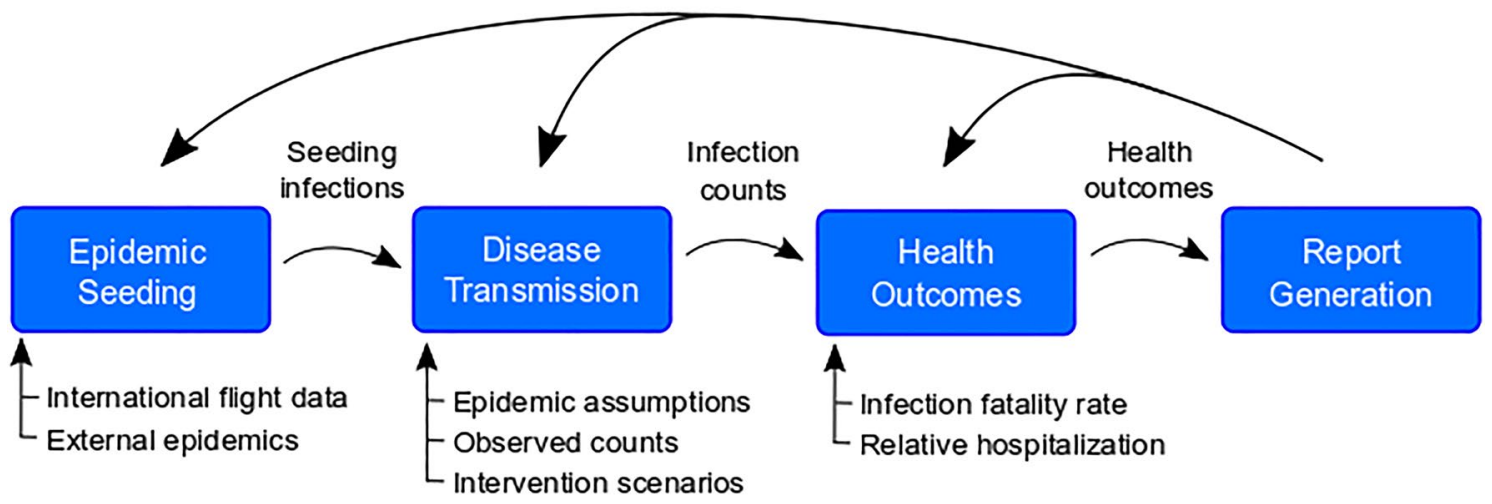

Figure 1. Overview of the pipeline. The pipeline has four modules, each with specific inputs that can be specified by the user. First, we identify when and in which model locations epidemics are seeded using an air importation model or confirmed case data. Second, the epidemic seeding events are used to initiate the disease transmission model, which is informed by our epidemiological assumptions and intervention scenarios. The disease transmission model produces daily incident infection counts and infection prevalence. Next, we calculate health outcomes like hospitalizations and ICU admissions from these infection counts according to assumptions about health outcome risks and infection fatality ratios. Finally, these health outcomes may be summarized using templates and functions from the report generation component of the pipeline.

\section{Methods}

Pipeline at a glance. The pipeline consists of multiple modular components designed to run in sequence to produce results and reports focused on policy relevant outcomes in any set of geographic locations (i.e., multiple countries, a single country, a set of subnational administrative units, or a single subnational administrative unit) (Fig. 1). While the pipeline was developed to be extended, the current core components are (1) epidemic seeding, (2) the transmission model, (3) health outcome generation engine, and (4) report generation.

These modular components of the pipeline fit together because each is composed of multiple pieces: an input format, an output format, one or more code libraries (where applicable), and a runner script. The standardized input and output formats ensure that components may be switched out according to user preference without impacting other phases. The "library" generally contains the core functions for a pipeline component. The script liaises between the user-defined configuration and the library by choosing which library to use and converting the input format to a format used by the library.

The pipeline runs these components in sequence, according to the specifications outlined in a configuration file. This makes it easy to add or modify a component. To add a component, we specify its input format, and incorporate its dependencies. To modify the implementation of a component, we add or modify functions in the library and function calls in the runner script. When appropriate, entire components may be substituted with data from outside of the pipeline, provided that the data meet the input formats required by the next pipeline phase.

Module 1: Epidemic seeding and initialization. "Epidemic seeding" refers to how the disease transmission module is initialized with infected individuals. A seeding module must produce one of more seeding files that specifies an added number of incident cases occurring due to "seeding" at particular dates and locations.

The pipeline currently contains two epidemic seeding options: (1) seeding according to first case appearance in data, and (2) seeding according to an air travel importation model.

Seeding according to earliest identified cases. This seeding option enables users to seed the model according to COVID-19 case data. It currently supports user-supplied data and downloads from two commonly used public sources, the Johns Hopkins University Center for Systems Science and Engineering (JHU-CSSE) COVID-19 Dashboard ${ }^{9}$ and USAFacts, a database that collates data from US state health departments ${ }^{10}$. Drawing from the user-specified data source, this option identifies the first five days that cases were reported in each modeled location. We assume that confirmed cases were infected a user-specified number of days prior to when they were reported, and that there is a user-specified ratio of infections to confirmed cases. Seed infections are, hence, created in each modeled location on the estimated days of infection for the first 5 days with reported cases; they are drawn stochastically from a Poisson distribution where the mean is the product of the number of reported cases and the user-specified ratio.

To facilitate the generation of the seeding file for US settings, we provide the "R/scripts/create_seeding.R" script in "HopkinsIDD/COVIDScenarioPipeline," which pulls data directly from USAFacts.

Seeding according to an air importation model. We adapted a previously published model of measles importation to model the rate of COVID-19 importation to specific locations due to air travel ${ }^{11}$. This seeding option, available in the Github repository "HopkinsIDD/covidImportation"12, uses complete itinerary (origin to final destination) air travel volume data from $\mathrm{OAG}^{13}$ for all airports in the world, source location populations, and 
source location incidence data, to inform a model with which absolute counts of importation are estimated on a daily basis into airports ${ }^{12}$.

Geographic areas surrounding airports are classified spatially into "airport catchment areas" with a Voronoi tessellation of space in reference to the latitude and longitude coordinates of the airport ${ }^{14}$. When there are multiple airports within close proximity, the user may specify a threshold distance under which airports may be grouped into a single cluster that is defined by its centroid. We then assign a probability of importation to each intersection of a Voronoi tile and an administrative unit boundary. This probability is calculated as the proportion of the airport catchment area population that lives in that intersection, assuming that population is distributed evenly by area. All air importations on a given day are then aggregated to the administrative unit level and seeded into the epidemic model as newly infected individuals.

Module 2: Transmission model and intervention scenarios. The disease transmission module takes in seeding information and produces an epidemic model output file that contains, at minimum for subsequent module compatibility, daily counts of incident infections, indexed to their time of symptom onset. The currently implemented default transmission module comprises a metapopulation model with stochastic SusceptibleExposed-Infected-Recovered (SEIR) disease dynamics.

Disease dynamics. The core model is a modified SEIR compartmental model where the time in the "Infected" compartment follows an Erlang distribution (i.e., the infected compartment is split into $k$ compartments) to produce more realistic infectious periods where the chance of recovery depends on the time since infection ${ }^{15}$, and a coefficient $(\alpha)$ can be set to help the model approximate non-homogeneous mixing between susceptible and infected individuals and non-exponential growth ${ }^{16}$. Currently $k$ is fixed at three compartments.

Transition of individuals between disease compartments is simulated stochastically with binomial random draws:

$$
\begin{gathered}
N_{S \rightarrow E}(t)=\operatorname{Binom}(S, 1-\exp (-\Delta t \cdot \mathrm{FOI}(\mathrm{t}))) \\
N_{E \rightarrow I^{(1)}}(t)=\operatorname{Binom}(E, 1-\exp (-\Delta t \cdot \sigma)) \\
\mathrm{N}_{\mathrm{I}^{(1)} \rightarrow \mathrm{I}^{(2)}}(\mathrm{t})=\operatorname{Binom}\left(\mathrm{I}^{(1)}, 1-\exp \left(-\Delta \mathrm{t} \cdot \gamma^{\prime}\right)\right) \\
N_{I^{(2)} \rightarrow I^{(3)}}(t)=\operatorname{Binom}\left(I^{(2)}, 1-\exp \left(-\Delta t \cdot \gamma^{\prime}\right)\right) \\
N_{I^{(3)} \rightarrow R}(t)=\operatorname{Binom}\left(I^{(3)}, 1-\exp \left(-\Delta t \cdot \gamma^{\prime}\right)\right) \\
\gamma^{\prime}=\gamma \cdot k
\end{gathered}
$$

where $S, E, I^{(1)}, I^{(2)}, I^{(3)}$, and $R$ represent the number of individuals in those respective compartments, $F O I(t)$ is the force of infection from the infected population on the susceptible population, $\frac{1}{\sigma}$ is the latent period, $\frac{1}{\gamma}$ is the infectious period, and $k$ is the number of $I$ compartments. The force of infection, which modulates transition of individuals from the $S$ to $E$ compartments:

$$
\begin{gathered}
\mathrm{I}(t)=\sum_{j=1}^{k} I^{(j)} \\
\mathrm{FOI}(t)=\beta \cdot \frac{I(t)^{\alpha}}{H} \\
\mathrm{H}=\mathrm{S}(t)+\mathrm{E}(t)+\sum_{j=1}^{k} I^{(j)}(t)+\mathrm{R}(t) \\
\beta(\mathrm{t})=\mathrm{R}_{0} \cdot \gamma
\end{gathered}
$$

where $H$ is the total population, $\beta$ is the daily transmission probability as defined by $R_{0}$ and the infectious period, and $\alpha$ is the mixing coefficient.

Metapopulation dynamics. The model is capable of simulating disease spread in multiple locations jointly according to assumptions about population mobility between individual model locations (e.g., administrative units). The SEIR disease dynamics described above are simulated in each model location with a modification to the force of infection term that accounts for this impact of mobility on disease spread.

The force of infection in a given location $i$ is calculated from a combination of local infections and infections in locations that are connected to it according to the mobility matrix, as follows: 


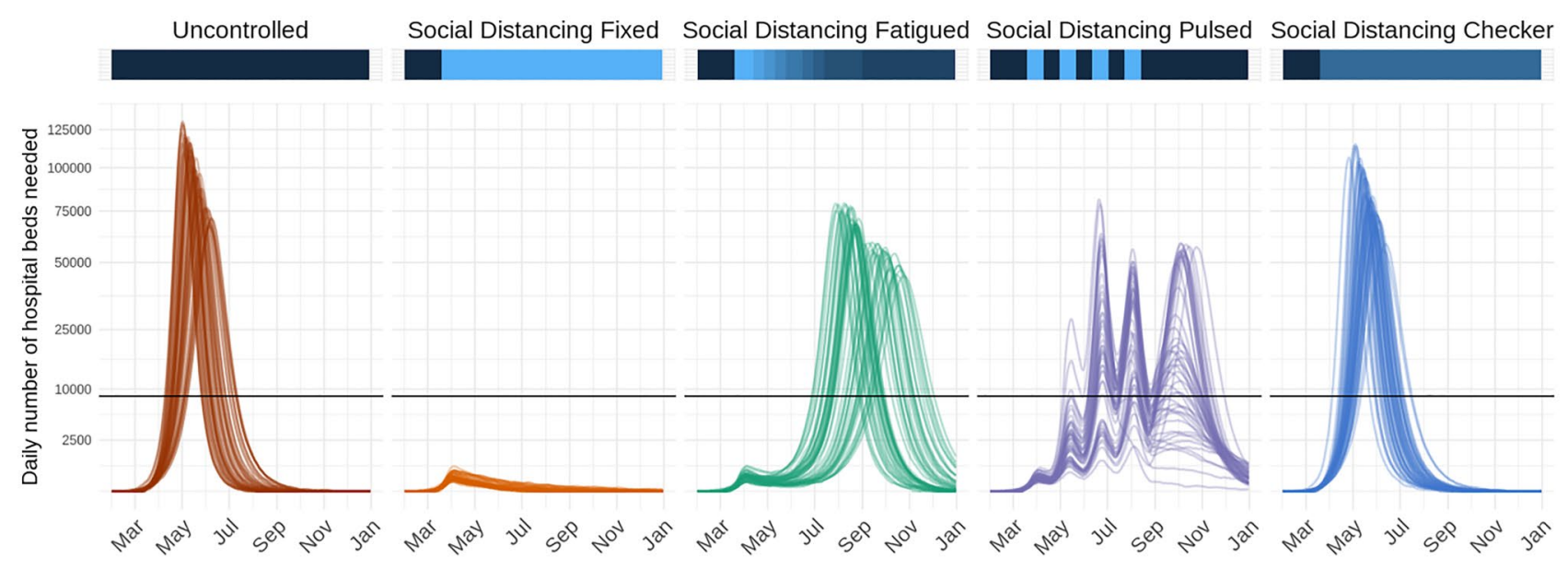

Figure 2. Time series of daily number of hospital beds needed across five possible intervention scenarios in a fictional location with nine counties. Lines represent results from 50 stochastic model simulations. Horizontal black lines represent the total hospital bed capacity in the fictional location, assumed to be $n=8661$ ( 3 beds per 1000 population). The colored horizontal bars along the top visualize the effectiveness of interventions at a given time point along a dark blue to light blue spectrum; dark blue indicates a period with no reductions to transmission, while light blue indicates a period with more restrictive action (i.e., low transmissibility). In the fifth scenario, "Social Distancing Checker," only three of nine counties implement any non-pharmaceutical interventions, thus differentiating it from "Social Distancing Fixed".

$$
\mathrm{FOI}_{i}(t)=\left(1-\sum_{j \neq i} p_{a} \frac{M_{i, j}}{H_{i}}\right) \cdot \beta_{i}(t) \frac{I_{i}(t)^{\alpha}}{H_{i}}+\sum_{j \neq i}\left(p_{a} \frac{M_{i, j}}{H_{i}} \cdot \beta_{j}(t) \frac{I_{j}(t)^{\alpha}}{H_{j}}\right)
$$

where $M$ is a mobility matrix such that $M_{i, j}$ represents the daily movement of individuals (e.g., commuting) from origin $i$ to destination $j, p_{a}$ is the proportion of time that moving individuals spend away, and $H_{i}$ is the population of node $i$. The transition of individuals between disease compartments may be modified to index by location $i$, for example:

$$
N_{S_{i} \rightarrow E_{i}}(t)=\operatorname{Binom}\left(S_{i}, 1-\exp \left(-\Delta t \cdot \mathrm{FOI}_{i}(\mathrm{t})\right)\right)
$$

Users may provide a symmetric or asymmetric wide-form mobility matrix for all model locations or a longform sparse mobility matrix that indicates only pairs of model locations with connectivity.

Application of transmission modifiers. In the absence of vaccines and other preventive treatments, non-pharmaceutical interventions, such as school closures, social distancing, stay-at-home directives, and testing and isolation are critical strategies for reducing disease transmission. Disease transmission may also change across space or time as the result of exogenous factors like seasonality or spatial heterogeneity in contact patterns. The model enables users to specify changes to the basic reproductive number $\left(R_{0}\right)$ and the inverse of the infectious period $(\gamma)$, for pre-specified periods of time to all or subsets of model locations independently. These interventions or exogenous changes can be implemented with fixed or distributional effectiveness. In addition, users may specify a rate of fatiguing effectiveness (e.g., declining adherence to a policy) over a certain number of days. This format enables flexibility in scenario planning; for instance, the model can be used to examine the effects of chaining multiple interventions together over time (e.g., school closure then stay-at-home), gradual declining adherence of the population to an intervention, switching interventions on and off over time, spatially heterogeneous interventions, or innate spatiotemporal heterogeneities (Fig. 2).

Transmission modifiers like non-pharmaceutical interventions or other exogenous factors modulate the daily transmission term below:

$$
\beta_{i}{ }^{\prime}(t)=\left(1-r_{i}(t)\right) \cdot \beta_{i}(t)
$$

where $\beta_{i}{ }^{\prime}(t)$ is the daily transmission rate after accounting for transmission modifier $r_{i}(t)$ at the specified location $i$ at time $t$. When transmission modifiers are in effect $\beta_{i}^{\prime}(t)$ replaces $\beta_{i}(t)$ in the force of infection term $\mathrm{FOI}_{i}(t)$.

The specification of transmission modifiers is completely user-specified. We include a set of common nonpharmaceutical intervention scenarios that can be applied for user-specified dates and locations (Table S1), which have been compiled according to our review of the literature on the potential impact of non-pharmaceutical interventions on respiratory virus transmission.

Module 3: Calculation of health outcomes. This pipeline module translates outputs from the transmission model into health outcomes such as hospitalizations and deaths. It takes in counts of daily incident infections and produces daily counts for specific health outcomes at appropriate time delays. 


\begin{tabular}{|l|l|}
\hline Column name (notation, as appropriate) & Description \\
\hline p_hosp_inf $\left(p_{\text {hosp|inf }}\right)$ & Probability of hospitalization among infected individuals \\
\hline p_icu_hosp $\left(\mathrm{p}_{\text {icu|hosp }}\right)$ & Probability of ICU admission among hospitalized individuals \\
\hline p_vent_icu $\left(p_{\text {vent } \mid i c u}\right)$ & Probability of ventilation among individuals in the ICU \\
\hline p_death_inf $\left(p_{\text {death } \mid \text { inf }}\right)$ & Probability of death among infected individuals \\
\hline rr_hosp_inf & Relative risk of hospitalization (given infection) relative to the average across all geoids \\
\hline rr_death_inf & Relative risk of death (given infection) relative to the average across all geoids \\
\hline
\end{tabular}

Table 1. Health outcome risk parameters.

The current default implementation produces hospital and intensive care unit (ICU) admissions, current hospital and ICU occupancy, ventilators needs, and number of deaths. Our modeling of health outcomes assumes that there is some transition probability from infection to death, infection to hospitalization, hospitalization to ICU admission, and ICU admission to ventilator use. In our modeling of health outcomes, we consider the probability of its occurrence (e.g., probability that infections are hospitalized), the time delay relative to its disease course (e.g., time between hospital admission and ICU admission), and where applicable, the duration in a given state (e.g., how long a patient remains ventilated).

For settings where hospitalization, ICU admission, and ventilator use is infrequent, it may be more appropriate to think of these health outcome projections as different levels of disease severity.

The user may specify health outcome probabilities and delays, conditional on the flows described above. For use as default values, we provide tables of parameter values which were derived from a literature review of COVID-19 health outcomes (Table S2).

The pipeline currently contains two versions of this module with different approaches to the specification of health outcome risks: (1) unadjusted, uniform risk and (2) location-specific risks, adjusted by key demographic or health factors in each location.

Unadjusted, population-wide health outcome risks. This option generates health outcome estimates with unadjusted risk across all locations, assuming fixed values for all health outcome probabilities, delays and durations.

We assumed that the number of infections admitted to the hospital is a draw from the Binomial distribution, lagged by a fixed time from symptom onset to hospital admission:

$$
n_{t_{\text {inf }}+t_{\text {inf } \rightarrow \text { hosp }}}^{\text {osp }} \sim \operatorname{Binom}\left(n_{t_{\text {inf }}}^{\text {inf }}, p_{\text {hosplinf }}\right)
$$

where $n^{\text {hosp }}$ is the number of hospital admissions, $n^{\text {inf }}$ is the number of infections, $t_{\text {inf }}$ is the time of infection, $t_{\text {inf } \rightarrow \text { hosp }}$ is the mean time delay between infection and hospital admission, and $p_{\text {hosplinf }}$ is the probability of hospitalization given infection (Table 1).

We make similar assumptions for the transitions between other outcomes (hospitalization admission to ICU admission, ICU admission to ventilator use, infection to death):

$$
\begin{gathered}
n_{t_{\text {hosp }}^{\text {icu }}+t_{\text {hosp } \rightarrow \text { icu }}}^{\text {Pinom }}\left(n_{t_{\text {hosp }}}^{\text {hosp }}, p_{\text {icu } \mid \text { hosp }}\right) \\
n_{t_{\text {icu }}+t_{\text {icu } \rightarrow \text { vent }}}^{\text {vent }} \\
n_{t_{\text {inf }}+t_{\text {inf } \rightarrow \text { death }}}^{\text {death }}
\end{gathered}
$$

The number of patients currently hospitalized, admitted to ICU, and ventilated are generated from the incident number of events and fixed (non-distributional) user-defined durations for each event.

As information about the death and hospitalization rates were scarce early on in the pandemic, decision makers wanted to consider how things would unfold over different scenarios of health burden. To facilitate these needs, our pipeline separates hospitalization and death rates from the other outcomes, and allows users to consider multiple scenarios for different rates.

Location-specific health outcome risks. The severity of disease from SARS-CoV-2 infection can vary greatly between locations due to difference between populations; individuals that are older, with limited access to health care, or with certain pre-existing health conditions are at greater risk for severe disease and death. For this reason, the hospitalization module also supports the specification of location-specific relative risks, which can be used, for example, to standardize hospitalization and mortality rates according to the age distribution of the population. Users may provide a wide format data file with standardized variable names for the transition probabilities and relative risks (columns) by geoid (rows). These transition probabilities are conditional on previous states (e.g., probability of hospitalization given infection is named p_hosp_inf). The probability of hospitalization and death given infection are specified as relative values compared to population-wide averages specified 
in the configuration file. We note that the location-specific standardizations apply only to the health outcomes, making a critical assumption that all individuals are at equal risk of infection.

While location-specific data is not required to differ for all health outcome transition probabilities (i.e., some probabilities can be constant across all locations), the location-specific file should include the variables described in Table 1 and population-wide average values for the probability of hospitalization (p_hosp_inf) and death (p_death_inf) given infection.

To facilitate construction of such files, we have created a companion package covidSeverity that produces location-specific relative death and hospitalization rates based on the age distribution of the local population ${ }^{17}$. The package generates these outputs for US counties based on data from the US Census Bureau, and we provide this as a model input file as part of the main pipeline implementation (see COVIDScenarioPipeline/ sample_data/geoid-params.csv). The package also includes built-in functionality to pull data from WorldPop ${ }^{18}$ and generate adjustments for any location of interest ${ }^{17}$.

The covidSeverity package applies a logistic generalized additive model (GAM) with a penalized cubic spline for age and random effect for age-specific estimates of risk of each health outcome from the literature, thus producing estimates of risk for 10-year, aggregated age categories. These age-specific estimates are then applied to the population age distribution in a given location.

Module 4: Summarization of model outputs. This component of the pipeline provides wrapper functions for the lightweight summarization of model outputs into quantiles, plotting functions for common figures, and R Markdown templates to facilitate the rapid generation of technical reports. This module is available in the R package report.generation in the Github repository "HopkinsIDD/COVIDScenarioPipeline."

We provide two key functions that read and process individual transmission (load_scenario_sims filtered) and health outcome (load hosp sims filtered) model output files. In managing individual files with these functions, we reduce the processing time and memory load. Both of these functions take processing functions as arguments, thus enabling aggregation and filtering to occur at the level of individual files.

The package contains technical report R Markdown templates for US states, US counties, and individual countries, a diagnostic report template called "sanity_check_report," and a template that is maintained solely for integration testing. When report.generation is installed and loaded, the templates become available to the user. Many parameters are drawn from the configuration file automatically and pre-written R Markdown chunks about the module options and methods can be referenced within the package.

Common figures include summary tables and time courses for estimated health outcomes under different interventions, maps that portray cumulative cases at the county level, comparisons between model estimates and observed cases and deaths, and visualizations for when ICU and ventilator capacity for each county is exceeded. The vignette below walks through example report outputs in more detail and we provide a template-generated report example in the "Supplementary Material" (Example Report).

Model specification. All components and settings for simulations from the COVID Scenario Pipeline model are specified in an easily-modifiable YAML configuration file. We describe different options in detail in the Results and an example of the complete set of configuration options is provided in the "Supplementary Material".

Model access and use. The project is open-source under the GNU General Public License v3.0 license, and code is available at https://github.com/HopkinsIDD/COVIDScenarioPipeline. The master branch of this repository consists of a Python package "SEIR" and two R packages "hospitalization" and "report.generation," which correspond to the second, third, and fourth modules of the model pipeline (https://zenodo.org/badge/ latestdoi/245866576). Air importation-based seeding is implemented in the covidImportation package (https:/github.com/HopkinsIDD/covidImportation), while seeding according to the earliest identified cases is performed in scripts within the "HopkinsIDD/COVIDScenarioPipeline" repository.

\section{Results: scenario modeling vignette}

Here, we present a vignette with 50 model simulations in a fictional setting (Location $\mathrm{X}$ ) with nine counties (named A through I) in demonstrative intervention scenarios from January 31 to December 31, 2020. In this vignette, we walk through how to set up and run the pipeline, demonstrate some of the spatial and temporal features for modeling non-pharmaceutical interventions, and display some of the plotting functions useful for summarizing the model output.

To run the model, users will require the functionality of the "HopkinsIDD/COVIDScenarioPipeline" repository and a second GitHub repository specific to their model location. A template for such a spatial repository may be found at "HopkinsIDD/COVID19_Minimal" (https://github.com/HopkinsIDD/COVID19_Minimal), and complete details on downloading and running the model are available in the template's wiki at https://github. com/HopkinsIDD/COVID19_Minimal/wiki.

Once we have a working environment, the next step is to create a configuration file to describe the model specifications. A skeleton configuration file is included in the COVID19_Minimal template, and the full configuration file accompanying the results of this vignette is in the "Supplementary Material".

First, we specify the broad parameters of the run, such as the date range covered and the number of simulations to run (see first section in "Supplementary Material", Example YAML Configuration File).

Next we provide spatial setup information identifying the location of files containing geographic data and the geographic targets for modeling. The spatial_setup section of the configuration file identifies a geographical data (geodata) file that contains the population data for each county or administrative subunit in the location(s) of interest and a mobility matrix file that contains the daily trip counts for each pair of counties. Users 
may employ the R scripts “build_US_setup.R” or "build_nonUS_setup.R," which are provided with the repository, to generate compatible geodata and mobility matrix files. For users modeling non-US settings, the Github repository "COVID-19-Mobility-Data-Network/mobility" can be used to fit real-time or sparse travel data with mobility models in order to generate similarly compatible mobility matrixes ${ }^{19,20}$.

We note that in situations with high cross-border mobility, it may be important to model a region larger than the location of interest in order to appropriately capture disease transmission risk in a given location. Here, we model Locations X, Y, and Z even though Location X is the sole location of interest:

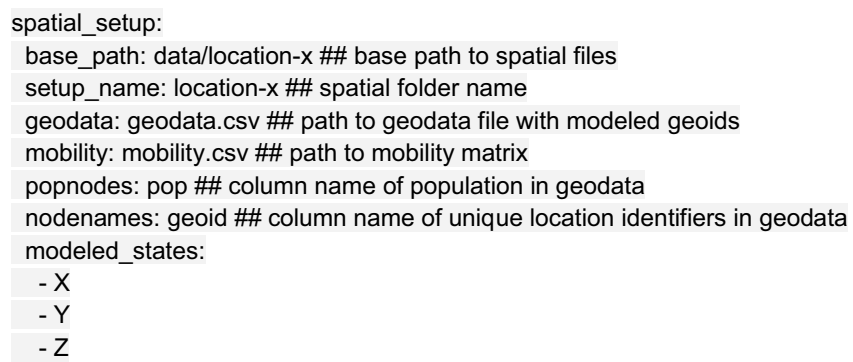

We then specify the locations of the seeding files (see seeding section in "Supplementary Material", Example YAML Configuration File), with a separate section to describe the air importation model parameters as appropriate (see importation in "Supplementary Material", Example YAML Configuration File).

Next, we specify the parameters that determine the course of the disease. The seir section of the configuration file defines parameters used in the SEIR disease transmission model, including the level of population mixing (where 1 is homogeneous mixing, and $<1$ is heterogeneous), the incubation period of the virus, the infectious period, and the baseline basic reproductive number $R_{0}$. These values may be fixed or drawn randomly from a distribution, according to the configuration file:

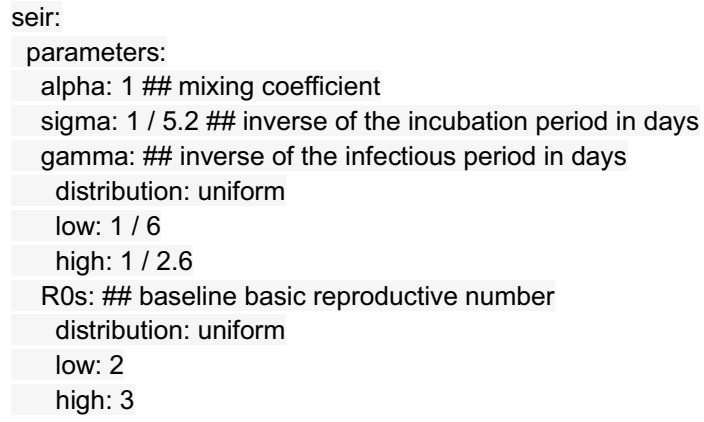

We typically parameterize sigma and gamma in our model with estimates of the range of the serial interval (SI) or generation time, such that

$$
\mathrm{SI}=\frac{1}{2}\left(\frac{1}{\gamma}\right)+\frac{1}{\sigma},
$$

which assumes that the average infection occurs halfway through an index case's infectious period.

The next step is defining the modeled intervention scenarios. Here, we considered five scenarios in our vignette example: (1) a no intervention scenario (named Uncontrolled), in which $R_{0}$ remains unchanged over the course of the outbreak; (2) social distancing measures with fixed effectiveness in place from March 19 to December 31 (SocialDistancing_fixed); (3) social distancing measures with declining compliance, which were modeled as $10 \%$ reductions in effectiveness every 2 weeks beginning March 19 (SocialDistancing_fatigued); (4) social distancing measures following a 3-week on-off "pulsing" cycle from March 19 to August 12 (SocialDistancing_pulsed); and (5) social distancing measures with spatial heterogeneity (SocialDistancing checker), where three of nine counties implement social distancing measures with fixed effectiveness from March 19 to December 31.

An intervention may be specified in a single block for all model locations, as in the case of the Socia 1Distancing_fixed scenario, or for unique location identifiers ("geoids") as in the SocialDistancing_checker scenario: 


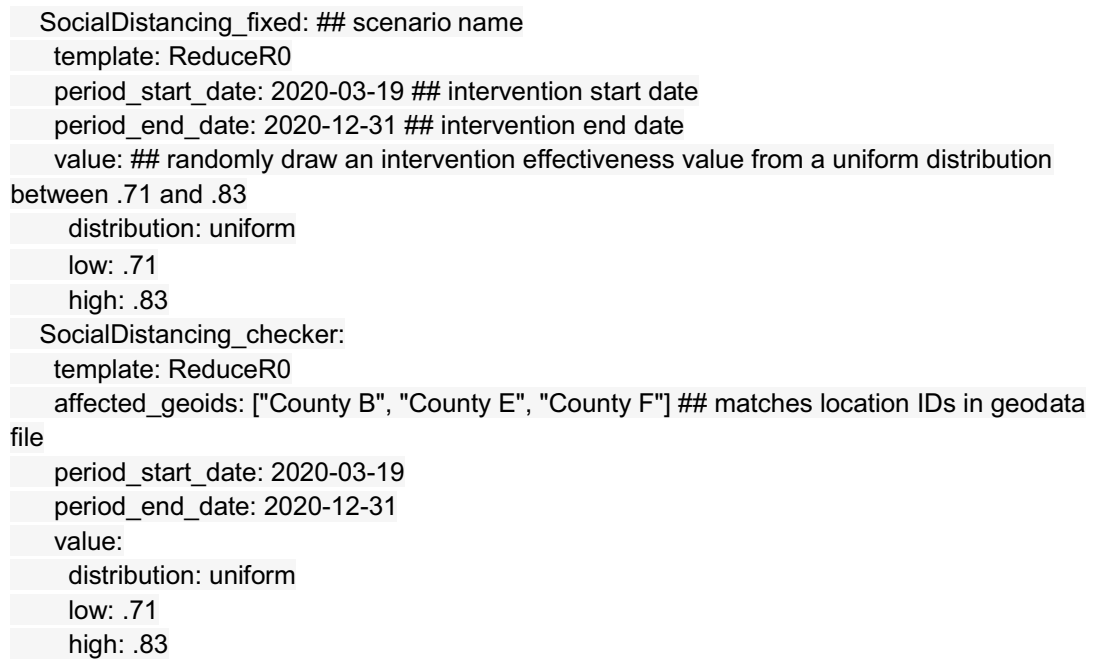

Other intervention scenarios require multiple blocks to be stacked together, as in the case of the SocialDistancing_pulsed scenario, shown in truncated form below:

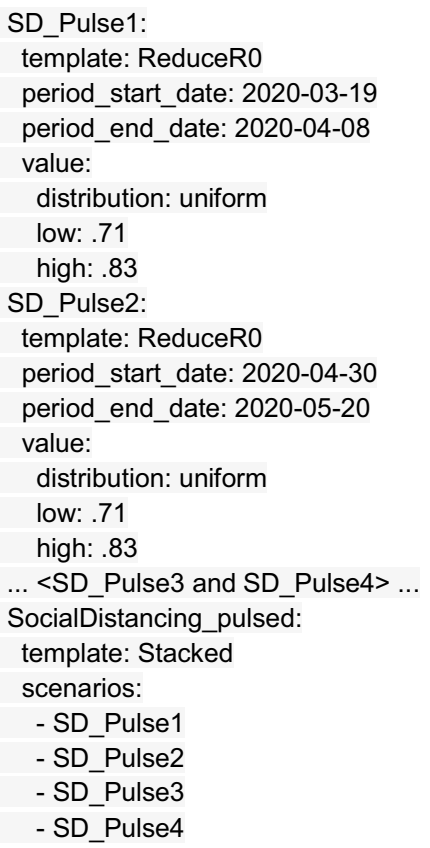

Then, we set up the health outcome risk specifications. In the hospitalization section of the configuration file, we specify whether the model calculates health outcome risks with age-adjusted estimates, the average infection fatality ratios (IFR), and the time delays between different health outcomes. The time delays are modeled with lognormal distributions and parameterized with the log median and log standard deviation. For ease of use, we provide a table of estimates found in the literature in Table S2.

After the simulation runs complete, we can produce rapid summaries of the results using R Markdown templates provided by the report. generation package. The report section of the configuration file specifies scenario labels and colors, IFR scenario labels, and table display dates. These settings, along with other model parameters, can be loaded into a technical report template.

Here we describe how the state_report template in the report.generation package can be used to display our model results as an example. The full template-generated report linked to this vignette is provided in the "Supplementary Material" (Example Report). First, the configuration file is loaded to pull in the model settings and file paths. Then, we use a number of predefined functions to load and plot the data.

For example, one standard report figure compares time series of the daily number of hospital beds needed across intervention scenarios (Fig. 2) using the load_hosp_geocombined_totals and plot_ts hosp_state_sample functions from the report.generation package in order to load and plot the 
data. To plot variations of these figures, we only need to change which health outcome variable is specified in plot ts hosp_state_sample.

While Fig. 2 displays aggregate results, our reports also provide location-specific risk and logistical outputs at the county- or administrative subunit-level. For example, we present the age-adjusted infection fatality ratios and risk of ICU admission among hospitalized infections for the modeled counties within the distribution of all counties in the United States in Fig. 3A,B. Using the load_hosp_geounit_relative_to_threshold and plot_needs_relative_to_threshold_heatmap functions from report.generation, we display the potential need for beds in excess of health system capacity by model location in our reports (Fig. 3C). Values of the location-specific healthcare capacity, represented by the number of staffed hospital, acute care, or intensive care beds available and/or number of ventilators available, are user-defined; these can be based on assumptions of the average availability per person or input from data where available.

A map of model outputs makes it easier to visualize spatial and temporal heterogeneity in different intervention scenarios (Fig. 4). We provide this functionality in the load_cum_inf_geounit_dates and plot geounit map functions in the report. generation package.

Each report template is equipped to load static $\mathrm{R}$ Markdown reference chunks, which we have pre-written and provided with the package. These chunks provide details on our methods, limitations, and key references, pulling in parameters from the configuration file as needed.

\section{Discussion}

We present our scenario pipeline as an open-source modeling framework that aims to balance epidemiological rigor with the flexibility and urgency required by public health policymaking. The modularity of our framework has enabled us to adapt our assumptions about COVID-19 epidemiology, transmission, and health outcome risks in response to emerging information and to different settings. The pipeline implementation of non-pharmaceutical interventions is highly adaptable for policymakers desiring to compare the impact of different potential scenarios.

Throughout the course of the pandemic, we have adapted the default settings of our pipeline in response to the changing needs and questions of our collaborators. At the beginning of the pandemic, air importation seeding was a critical determinant of epidemic onset in specific locations. Now that cases of COVID-19 are present worldwide, we have shifted towards developing more empirical methods of epidemic seeding that better match trajectories of confirmed cases in specific locations as policy questions have shifted to more operational needs. As new data emerged, we moved from calculating unadjusted health outcomes to health outcomes based on age-standardized risk of hospitalization, ICU admission, and death according to emerging case-study data.

As the COVID-19 pandemic continues, we plan to continue expanding the scope of the COVID Scenario Pipeline to changing needs and questions. Future model releases will include a health outcomes model expansion that will enable a multiplicity of pathways to ICU occupancy, ventilator usage, and death. As questions have shifted to near-term operational needs, we have also begun to incorporate inference into our models, thus enabling the calibration of model trajectories to deaths and confirmed case counts, short-term forecast of health outcomes, and estimation of location-specific transmission parameters and NPI effectiveness.

While the pipeline's generic structure means that different modules may be readily replaced, the current implementation of our model has several limitations with regard to the epidemiology of COVID-19. The disease transmission model does not explicitly incorporate age-specific contact or transmission rates or asymptomatic transmission, nor does it consider how factors like testing rates may lead to time-varying biases in reporting. Data to inform these factors was scarce when this model was initially developed, but these processes can change the dynamics displayed by scenario projections. Moreover, this model structure means that we cannot model age-targeted interventions (e.g., cocooning of high-risk age groups) or interventions targeting asymptomatic individuals (e.g., systematic age-specific testing) in a mechanistic manner. However, we can and do adjust for these types of interventions through overall population-level reductions in disease transmission. Additionally, the current model cannot tune changes in population mobility or connectivity over time, although we know that travel and movement restrictions played a role in changing the spread of COVID-19.

The health outcomes module also has several structural limitations; we assume that only one progression in health outcome severity exists (infection to hospitalization to ICU to ventilator use), although we know that many disease course progressions are possible. In addition, the delays and durations involved in our health outcomes progression are fixed in time for a given location, although stochastic variation may be incorporated across simulations..

Nevertheless, the modular approach taken is meant to allow for easy substitution of models with improvement in any of these areas while still taking advantage of other pipeline components. We have leveraged this feature throughout the course of the COVID-19 pandemic, and individual modules continue to develop. This flexibility does come at a cost, as the modular pipeline approach requires us to write and read files at the end and beginning of each phase, respectively. This procedure requires more disk space and input/output steps than other modeling approaches that can hold all of the necessary data in memory until a single output is produced at the end. Still, these slowdowns are not critically limiting; we have been able to run 1000 county-level simulations of the United States in less than 10 min on a 96 core server.

These limitations point to a broader need to consider the totality of evidence generated by epidemiologic models. While our approach is well-suited to answering policy questions about interventions, it is critical for policymakers to explore projections from multiple models in order to understand the range of possible trajectories and the sensitivity of results to different assumptions. Models that incorporate individual-level behaviors may be better for considering the impact of specific contact tracing strategies or location-specific measures like workplace occupancy or symptom screening policies that are not well captured in compartmental models such 
A

A

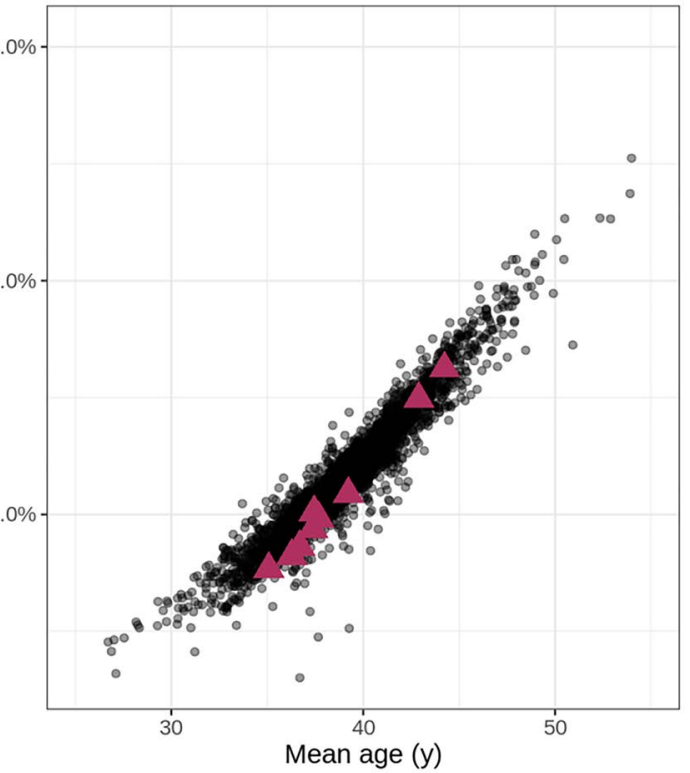

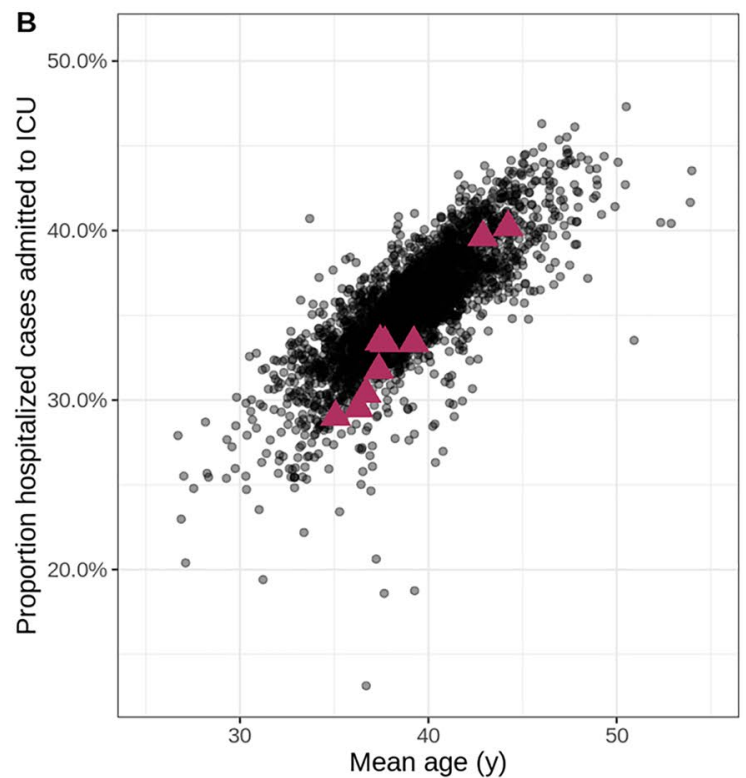

C
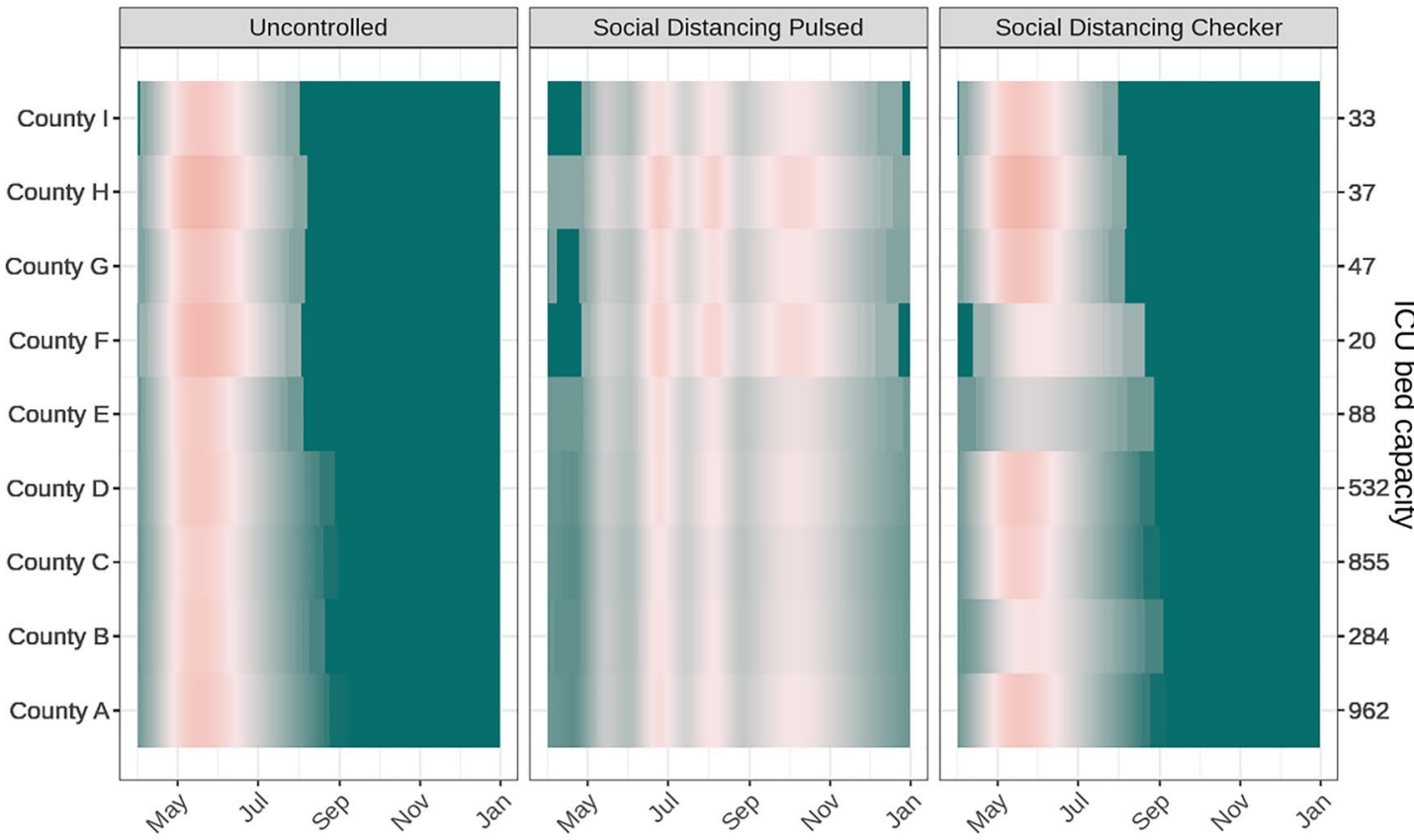

Log excess ICU beds needed

$-5.0-2.50 .0$

Figure 3. Health outcome risks and logistical needs for fictional counties. In the scatterplots, each point indicates (A) the age-adjusted infection fatality ratio and (B) risk of ICU admission given hospitalization by mean age for a county within the United States. Data for the nine fictional counties in our vignette is marked by magenta triangles. (C) The heat maps display county-level ICU bed needs, shaded according to the log ratio above or below the assumed ICU bed capacity (secondary y-axis) in each county (primary y-axis) for three example intervention scenarios (panels). The salmon pink shading indicates periods of time where ICU bed needs exceed capacity in the fictional counties.

as ours ${ }^{21-23}$. Other individual-based models are better suited for addressing heterogeneities due to differences in household or social structures ${ }^{24,25}$. Models incorporating real-time mobility data can best characterize the impact of movement-related restriction ${ }^{26}$ models with age-specific transmission may provide more detail on the impact of age-specific interventions like "cocooning" ${ }^{27}$ or closing and opening schools". Still other models are particularly suited to address questions about health systems burden and forecast operational need $s^{5,28,29}$ and to consider the economic impacts of transmission and interventions ${ }^{30,31}$. Integrating knowledge from multiple models, where appropriate, with careful consideration of the assumptions and appropriate applications of each model, will strengthen response and preparedness ${ }^{32}$. 
A Uncontrolled: Jun 01

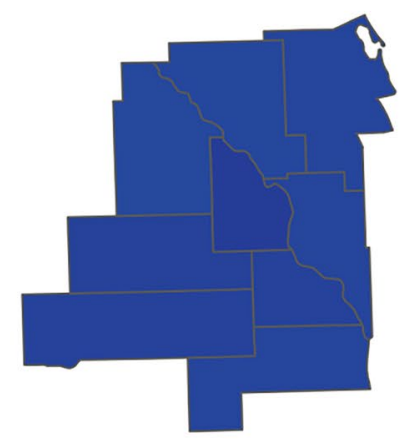

D

Uncontrolled: Jun 01

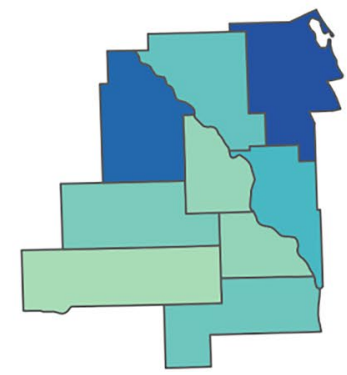

B

Social Distancing Pulsed: Jun 01

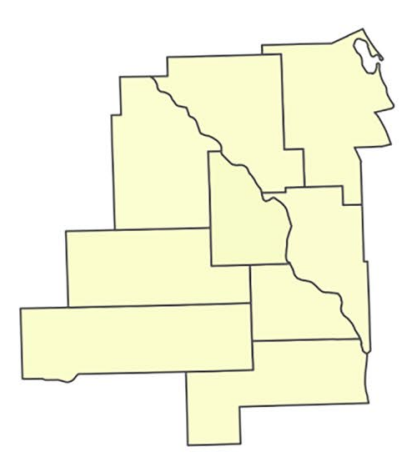

E

Social Distancing Pulsed: Jun 01

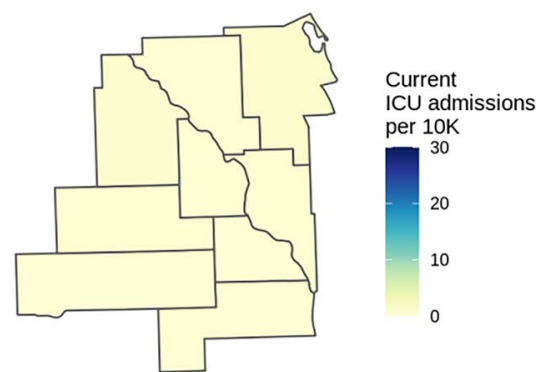

C

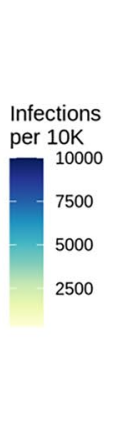

$\mathrm{F}$

Social Distancing Checker: Jun 01

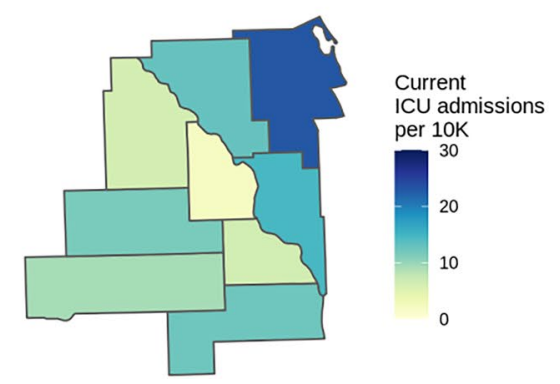

Figure 4. County-level COVID-19 risk for three scenarios (Uncontrolled, Pulsed, Checker) in the fictional Location X. Choropleths for model outcomes on June 1, 2020 of the (A-C) cumulative infection rate per 10,000 population and (D-F) number of patients currently admitted to the ICU per 10,000 population for the Uncontrolled, Social Distancing Pulsed, and Social Distancing Checker intervention scenarios. Countylevel variation in attack rates can arise from differences in risk of importation, mobility patterns connecting subdivisions, and differences in non-pharmaceutical interventions applied in each location. If location-specific health outcome risks are specified (as are age-standardized health outcome risks in this example), this may serve as another source of county-level variation.

Our flexible modeling pipeline brings an important voice to this "conversation" of models, by allowing rapid and flexible specification and simulation of even very complex intervention scenarios, and providing flexibility to rapidly update models as our understanding of a disease changes. This approach only reaches its full potential when parameters are based on careful and ongoing consideration of the literature and available data. But, when appropriately used as part of an iterative approach to decision making, this pipeline can be a valuable tool for public health decision making.

Received: 9 September 2020; Accepted: 18 March 2021

Published online: 06 April 2021

\section{References}

1. Zhu, N. et al. A novel coronavirus from patients with pneumonia in China, 2019. N. Engl. J. Med. 382, 727-733 (2020).

2. WHO Director-General's opening remarks at the media briefing on COVID-19-11 March 2020. in World Health Organization [Internet]. Accessed 11 Mar 2020 [cited 22 May 2020]. https://www.who.int/dg/speeches/detail/who-director-general-s-openi ng-remarks-at-the-media-briefing-on-covid-19---11-march-2020.

3. Huang, C. et al. Clinical features of patients infected with 2019 novel coronavirus in Wuhan, China. Lancet 395, 497-506 (2020).

4. Ferguson, N., Laydon, D., Gilani, G.N., Imai, N., Ainslie, K., Baguelin, M., et al. Report 9: Impact of non-pharmaceutical interventions (NPIs) to reduce COVID19 mortality and healthcare demand. 2020 [cited 22 May 2020]. https://doi.org/10.25561/77482.

5. Branas, C.C., Rundle, A., Pei, S., Yang, W., Carr, B.G., Sims, S., et al. Flattening the curve before it flattens us: Hospital critical care capacity limits and mortality from novel coronavirus (SARS-CoV2) cases in US counties. medRxiv (2020) https://doi.org/10.1101/ 2020.04.01.20049759.

6. Moghadas, S. M. et al. Projecting hospital utilization during the COVID-19 outbreaks in the United States. Proc. Natl. Acad. Sci. USA 117, 9122-9126 (2020).

7. Davies, N.G., Klepac, P., Liu, Y., Prem, K., Jit, M., CMMID COVID-19 Working Group, et al. Age-dependent effects in the transmission and control of COVID-19 epidemics. Nat Med 26, 1205-1211 (2020). https://doi.org/10.1038/s41591-020-0962-9.

8. IHME COVID-19 Health Service Utilization Forecasting Team, Murray, C.J.L. Forecasting the impact of the first wave of the COVID-19 pandemic on hospital demand and deaths for the USA and European Economic Area countries. medRxiv (2020) https:// doi.org/10.1101/2020.04.21.20074732. 
9. Dong, E., Du, H. \& Gardner, L. An interactive web-based dashboard to track COVID-19 in real time. Lancet Infect Dis. 20, 533-534 (2020).

10. "US COVID-19 cases and deaths by state" in USA Facts [Internet]. [cited 23 May 2020]. usafacts.org.

11. Truelove, S.A., Mier-y-Teran-Romero, L., Gastanaduy, P., Taylor Walker, A., Berro, A., Lessler, J., et al. Epidemics, air travel, and elimination in a globalized world: The case of measles. medRxiv (2020) https://doi.org/10.1101/2020.05.08.20095414.

12. Truelove, S., Lauer, S.A., Lemaitre, J., Kaminsky, K., Kaminsky, J. HopkinsIDD/covidImportation: Initial release of covidImportation R package. (2020). [cited 23 May 2020]. https://doi.org/10.5281/zenodo.3840560.

13. Flight data in OAG [Internet]. [cited 23 May 2020]. oag.com.

14. Balcan, D. et al. Modeling the spatial spread of infectious diseases: The GLobal Epidemic and Mobility computational model. J. Comput. Sci. 1, 132-145 (2010).

15. Yan, P. \& Chowell, G. Quantitative methods for investigating infectious disease outbreaks. Texts Appl. Math. https://doi.org/10. 1007/978-3-030-21923-9 (2019).

16. Finkenstädt, B. F., Biørnstad, O. N. \& Grenfell, B. T. A stochastic model for extinction and recurrence of epidemics: Estimation and inference for measles outbreaks. Biostatistics 3, 493-510 (2002).

17. Lauer, S.A., Truelove, S.A., Grantz, K. HopkinsIDD/covidSeverity: Initial release of covidSeverity R package. (2020) [cited 23 May 2020]. https://doi.org/10.5281/zenodo.3840716.

18. "Population counts" in WorldPop [Internet]. [cited 24 May 2020]. https://www.worldpop.org/.

19. Giles, J. COVID-19-Mobility-Data-Network/mobility: v0.1.1 alpha release of mobility R package. (2020) [cited 23 May 2020]. https://doi.org/10.5281/zenodo.3838719.

20. Giles, J. and Wesolowski, A. mobility: an R package for modeling human mobility patterns. [cited 23 May 2020]. https://covid-19mobility-data-network.github.io/mobility/index.html.

21. Kucharski, A. J. et al. Effectiveness of isolation, testing, contact tracing, and physical distancing on reducing transmission of SARSCoV-2 in different settings: A mathematical modelling study. Lancet Infect. Dis. 20, 1151-1160 (2020).

22. Firth, J. A. et al. Using a real-world network to model localized COVID-19 control strategies. Nat. Med. 26, 1616-1622 (2020).

23. Hinch, R., Probert, W.J.M., Nurtay, A., Kendall, M., Wymatt, C., Hall, M., et al. OpenABM-Covid19-An agent-based model for non-pharmaceutical interventions against COVID-19 including contact tracing. medRxiv (2020) https://doi.org/10.1101/2020. 09.16.20195925.

24. Wilder, B. et al. Modeling between-population variation in COVID-19 dynamics in Hubei, Lombardy, and New York City. Proc. Natl. Acad. Sci. USA 117, 25904-25910 (2020).

25. Kerr, C. C. et al. Covasim: An agent-based model of COVID-19 dynamics and interventions. medRxiv (2020) https://doi.org/10. $1101 / 2020.05 .10 .20097469$

26. Lai, S., Ruktanonchai, N.W., Zhou, L., Prosper, O., Luo, W., Floyd, J.R., et al. Effect of non-pharmaceutical interventions to contain COVID-19 in China. Nature 585, 410-413 (2020).

27. Duque, D., Morton, D.P., Singh, B., Du, Z., Pasco, R., Meyers, L.A. COVID-19: How to relax social distancing if you must. medRxiv (2020) https://doi.org/10.1101/2020.04.29.20085134.

28. Los Alamos National Laboratory. COVID-19 Cases and Deaths Forecasts. [cited 24 May 2020]. https://covid-19.bsvgateway.org/.

29. Weissman, G. E. et al. Locally hospital informed simulation to predict capacity needs during the COVID-19 pandemic. Ann. Intern. Med. 173, 21-28 (2020).

30. Acemoglu, D., Chernozhukov, V., Werning, I., Whinston, M. D. Optimal targeted lockdowns in a multi-group SIR model. National Bureau of Economic Research, Working Paper 27102 (2020). http://www.nber.org/papers/w27102.

31. Silva, P. C. L. et al. COVID-ABS: An agent-based model of COVID-19 epidemic to simulate health and economic effects of social distancing interventions. Chaos Solitons Fractals. 139, 110088 (2020).

32. Shea, K. et al. Harnessing multiple models for outbreak management. Science 368, 577-579 (2020)

\section{Acknowledgements}

We acknowledge technical support from Amazon Web Services, with particular thanks to Pierre-Yves Aquilanti, Karthik Raman, Arun Subramaniyan, Greg Thursam, and Anh Tran for their time and expertise.

\section{Author contributions}

K.H.G., J.K., H.R.M., S.A.T., L.T.K., J.L., and E.C.L. wrote the first draft of the manuscript. J.C.L., J.K., K.H.G., and S.A.T. wrote the core model code. K.H.G. and H.R.M. prepared the manuscript figures. H.R.M. prepared the supplementary material. S.S., J.W., K.K., and J.P.S. contributed to model and code optimization and documentation. J.L. and E.C.L. guided the design, analysis, and execution of the project. J.C.L., K.H.G., J.K., H.R.M., S.A.T., S.A.L., L.T.K., J.P.-S., J.L., and E.C.L. contributed to study design and analysis. All authors contributed model code and reviewed the manuscript.

\section{Funding}

JCL, KHG, JK, HRM, SAT, SAL, LTK, JPS, JL, and ECL were supported by the State of California. JCL, JK, SAT, SAL, LTK, JPS, JL, and ECL were supported by the US Department of Health and Human Services and the US Department of Homeland Security. JCL acknowledges funds provided by the Swiss National Science Foundation (200021-172578) and the attached mobility Grant. SAT acknowledges funds provided by the US Office of Foreign Disaster Assistance (130492) and the Centers for Disease Control and Prevention (126280). LTK acknowledges funds provided by the Centers for Disease Control and Prevention (5U01CK000538-03) and the University of Utah Immunology, Inflammation, \& Infectious Disease Initiative (26798 Seed Grant). This work was also supported with computing service credits from Amazon Web Services and more generally by the Johns Hopkins Health System and the Office of the Dean at the Johns Hopkins Bloomberg School of Public Health.

\section{Competing interests}

The authors declare no competing interests.

\section{Additional information}

Supplementary Information The online version contains supplementary material available at https://doi.org/ 10.1038/s41598-021-86811-0.

Correspondence and requests for materials should be addressed to E.C.L. 
Reprints and permissions information is available at www.nature.com/reprints.

Publisher's note Springer Nature remains neutral with regard to jurisdictional claims in published maps and institutional affiliations.

(c) (i) Open Access This article is licensed under a Creative Commons Attribution 4.0 International License, which permits use, sharing, adaptation, distribution and reproduction in any medium or format, as long as you give appropriate credit to the original author(s) and the source, provide a link to the Creative Commons licence, and indicate if changes were made. The images or other third party material in this article are included in the article's Creative Commons licence, unless indicated otherwise in a credit line to the material. If material is not included in the article's Creative Commons licence and your intended use is not permitted by statutory regulation or exceeds the permitted use, you will need to obtain permission directly from the copyright holder. To view a copy of this licence, visit http://creativecommons.org/licenses/by/4.0/.

(C) The Author(s) 2021 